\title{
Traitements ethno-vétérinaires des parasitoses digestives des petits ruminants dans le plateau central du Burkina Faso
}

\author{
Adama KABORE ${ }^{1^{*}}$, Hamidou H. TAMBOURA ${ }^{1}$, Adrien Marie Gaston BELEM ${ }^{2}$ et \\ Amadou TRAORE ${ }^{1}$ \\ ${ }^{1}$ Institut de l'Environnement et de Recherches Agricoles (INERA) /Département Productions Animales (DPA), \\ 01 BP 476 Ouagadougou / Burkina Faso, Tél.: (226) 50319229 Fax : (226) 50340271. \\ ${ }^{2}$ Institut du Développement Rural (IDR)/Université Polytechnique de Bobo-Dioulasso, 01 BP 3770 \\ Ouagadougou 01, Burkina Faso. \\ *Auteur correspondant, E-mail: ade1_bf@yahoo.fr
}

\section{RESUME}

La présente étude visait d'une part, à mieux comprendre les motivations des éleveurs à recourir aux méthodes traditionnelles de soins pour leurs animaux, et d'autre part, à recenser les remèdes utilisés dans la région du plateau central du Burkina Faso. Elle s’est déroulée de juin à octobre 2006 en deux étapes : i) une phase de groupe (focus-group screening) avec un formulaire-guide d'entretien comme support d'interview semi-structurées (ISS) auprès de 101 éleveurs et ii) une phase individuelle sous la forme d'enquêtes formelles avec un formulaire administré successivement à 21 tradipraticiens vétérinaires reconnus compétents dans le traitement des parasitoses digestives des petits ruminants par la communauté des éleveurs. Les résultats ont montré que 50.5\% des éleveurs utilisaient concomitamment la médecine vétérinaire moderne et celle traditionnelle pour soigner les animaux malades. Les tradipraticiens vétérinaires qui prestent pour ces éleveurs sont âgés en moyenne de 57,6 ans et sont analphabètes. Les remèdes traditionnels proposés pour lutter contre les parasites digestifs des petits ruminants de la région sont essentiellement à base de plantes locales $(88,8 \%)$ composées de douze (12) espèces appartenant à neuf (9) familles botaniques. Leurs modes de préparation et d'administration aux animaux sont décrits selon les dires des tradipraticiens enquêtés.

(C) 2007 International Formulae Group. All rights reserved.

Mots clés: Pharmacopée vétérinaire; parasites digestifs; petits ruminants; région centrale, Burkina Faso.

\section{INTRODUCTION}

Au Burkina Faso $\left(10^{\circ}\right.$ et $15^{\circ}$ de latitude nord, $2^{\circ}$ de longitude est et $5^{\circ} 30^{\prime}$ de longitude ouest), pays sahélien situé au cœur de l'Afrique de l'Ouest, l'élevage tient une place importante dans l'économie nationale. En effet, ce secteur d'activité occupe près de $80 \%$ de la population rurale active du pays et contribue à hauteur de $26 \%$ aux recettes d'exportation et $10 \%$ au Produit Intérieur Brut (INERA, 2002).

Malheureusement son mode d'exploitation est dominé par un système traditionnel confronté aux pathologies dues aux parasitoses digestives qui réduisent l'expression de la productivité des animaux en général et celles des petits ruminants en particulier (Krecek et Waller, 2006). Ces maladies constituent ainsi des sources de grande préoccupation constante pour les producteurs ruraux qui ne disposent pas de revenus financiers suffisants pour assurer une bonne couverture médicale. Pour minimiser ces effets, des solutions alternatives endogènes sont de plus en plus explorées par ces producteurs pour lesquels l'élevage des petits ruminants (ovins et caprins) est une source de sécurité alimentaire, de revenus monétaires et de raffermissement de la cohésion sociale. Il s'agit en l'occurrence de la pharmacopée vétérinaire traditionnelle qui constitue un recours très important pour lutter 
contre ces maladies animales (Tamboura et al., 1998; CAPES, 2006).

La présente étude vise à recenser les remèdes traditionnels prescrits par les tradithérapeutes du plateau central du Burkina Faso pour lutter contre les parasitoses digestives des petits ruminants et de proposer des formes de valorisation qui restent à la portée des producteurs.

\section{MATERIEL ET METHODES Milieu d'étude}

L’étude s'est déroulée de juin à octobre 2006, dans 38 villages des provinces du Bazèga, chef lieu Kombissiri (1204' de latitude nord et $1^{\circ} 34^{\prime}$ de longitude ouest) et du Kadiogo, chef lieu Ouagadougou (12³5' de latitude nord et $1^{\circ} 52^{\prime}$ de longitude ouest). Le climat de la zone est de type nord-soudanien avec une pluviométrie annuelle moyenne qui varie entre 600 et $900 \mathrm{~mm}$. Il est caractérisé par l'alternance d'une saison de pluies de 4 à 5 mois (juin à octobre) et d'une saison sèche de 7 à 8 mois. La température ambiante présente de grandes variations avec des moyennes entre $15^{\circ} \mathrm{C}$ et $30^{\circ} \mathrm{C}$ en décembre et janvier, et entre $30^{\circ} \mathrm{C}$ et $45^{\circ} \mathrm{C}$ pendant les mois de mars à mai. La végétation est constituée de savanes arborées à arbustives comprenant des essences ligneuses comme Parkia, Combretum, Piliostigma ... et des herbacées du genre Andropogon, Pennisetum , Brachiaria, Cenchrus, ...

\section{Méthodologie}

Des enquêtes ethno-vétérinaires ont été réalisées successivement à deux niveaux selon la procédure décrite précédemment par Tamboura et al. (1998). Brièvement, une enquête préliminaire a été réalisée auprès de 101 éleveurs de la zone dans une $1^{\text {ere }}$ étape. Ces éleveurs ont été choisis sur la base de la possession d'animaux (notamment de petits ruminants) et de la disponibilité à participer à l'enquête. Il s'agissait d'obtenir des informations précises sur les pratiques d’élevage, les soins de santé animale, les motivations individuelles du recours aux remèdes traditionnels et les principales maladies animales rencontrées dans la région. A l'issue de cette étape, chaque enquêté participait à l'identification de personnesressources (tradipraticiens vétérinaires) bien connues présentes dans le terroir et auxquelles ils font recours en cas de besoins pour les soins traditionnels. Ces personnes-ressources ont constitué le $2^{\text {ème }}$ échantillon qui a servi pour l'étape suivante. Ainsi, pour la $2^{\text {ème }}$ étape, 21 tradipraticiens vétérinaires reconnus par les éleveurs comme étant spécialisés dans le traitement des maladies animales (spécialement les parasitoses digestives des petits ruminants) ont fait l'objet d'enquêtes formelles. Les informations recueillies à leur niveau ont porté sur leur profil socioéconomique, leur connaissance des parasitoses des ruminants et l'identification des plantes médicinales utilisées pour le traitement de ces pathologies (identification, description, techniques de préparation, modes d'administration et mode de conservation des remèdes). La reconnaissance botanique de toutes les plantes proposées par les tradipraticiens s'est faite à partir de l'herbier du laboratoire de botanique appliquée du Département de Productions Forestières au Centre National de Recherches Scientifiques et Techniques (CNRST) où sont déposés des spécimens.

Deux types de questionnaires ont ainsi été utilisés à raison d'un par étape, après que chaque fiche ait été testée puis validée au préalable.

\section{Analyse statistique}

Les données collectées ont été dépouillées manuellement avant d'être codifiées et saisies avec le logiciel Excel, version 4.0 de Windows 2003. Ensuite, elles ont été soumises à un calcul de moyennes et de fréquences avec le logiciel SPSS (version 10.0.5).

\section{RESULTATS}

\section{Le degré et les motivations du recours à la} médecine traditionnelle vétérinaire

Les résultats obtenus montrent que l'ensemble des éleveurs enquêtés a recours à la médecine moderne pour soigner leurs animaux malades. Les principaux anthelminthiques conventionnels utilisés sont Synanthic bolus ${ }^{\circledR}$ (oxfendazole), Benzal ${ }^{\circledR}$ (albendazole), Bolumizole ${ }^{\circledR}$ (levamisole) et Ivomec $^{\circledR}$ (ivermectine). Toutefois, la moitié d'entre eux (50,5 \%) affirme recourir également à la médecine vétérinaire traditionnelle. Pour cela, quatre principales raisons sont évoquées. Il s’agit de l’absence 
ou de l'indisponibilité de l’agent vétérinaire au moment opportun pour soigner les animaux malades (66,3\% de cas exprimés), de la cherté des médicaments conventionnels (66,3\%), de l'efficacité reconnue aux remèdes traditionnels $(23,7 \%)$ et enfin des cas de plus en plus fréquents de l'inefficacité de certains traitements modernes (9,9\%).

\section{Les tradipraticiens vétérinaires et leurs pratiques de traitement}

Parmi les vingt un (21) tradipraticiens vétérinaires identifiés par les éleveurs, 76\% (composés d'autant d'ethnie peul que d'ethnie mossi) ont accepté de se soumettre aux questionnaires. Ils sont tous soit des éleveurs purs, soit des agro-pasteurs. Aucun d'entre eux n'est alphabétisé et l'échantillon ne comporte pas de femme tradipraticienne. L’âge des personnes interrogées varie de 40 à 86 ans avec une moyenne de 57,6 \pm 11,0 ans.

\section{Étiologie, épizootiologie et diagnostic du parasitisme des ruminants}

Selon les tradipraticiens interrogés, les principaux facteurs favorisant l'apparition des parasites digestifs chez les ruminants sont les eaux sales des mares et des retenues d'eau (56,2\% des réponses), les herbes souillées des zones de pâture (50\%) et les pâturages des bas fonds (12,5\%). Ces éléments sont reconnus favorables à l'apparition et/ou à l'accentuation de la prévalence de l'infestation des ruminants.

Traditionnellement, le diagnostic de la maladie se fonde sur les symptômes cliniques observés sur les animaux malades. Les principaux signes cliniques que décrivent les tradipraticiens sont par ordre décroissant la présence de vers dans les fecès $(62,5 \%)$, l'amaigrissement (50\%), les poils piqués (31,5\%), l'anorexie (25\%) et la diarrhée $(18,7 \%)$. Ces signes sont observables en toute saison, avec une prédominance en saison des pluies, notamment chez les jeunes animaux. En outre, ces signes ne sont pas exclusifs les uns des autres, avec un tableau de concomitance de deux ou plusieurs signes à la fois $(62,5 \%$ des cas). Le trinôme le plus fréquemment observé est « amaigrissement/vers dans les fecès/anorexie » (25\%), suivi des binômes «vers dans les fecès/amaigrissement »
(31,5\%), «poils piqués/amaigrissement» $(18,7 \%)$ et enfin «poils piqués/vers dans les fecès » $(12,5 \%)$.

Remèdes traditionnels contre le parasitisme digestif des petits ruminants

Dix huit (18) remèdes ont été prescrits par les tradipraticiens vétérinaires comme étant efficaces contre les parasitoses digestives des petits ruminants. Le tableau 1 présente ces remèdes. Du point de vue analytique, ils sont composés essentiellement de végétaux $(88,8 \%)$ et sont généralement préparés extemporanément au moment de l'utilisation. Ils sont généralement monospécifiques (93,7\%). D’autres ingrédients sont parfois ajoutés comme le sel, la potasse, la poudre de charbon végétal, etc. Sur l'ensemble, on a identifié 12 espèces végétales appartenant à 9 familles botaniques. Anogeissus leiocarpus a été la plante la plus citée par les prescripteurs $(27,7 \%)$, suivie de Khaya senegalensis $(16,6 \%)$ et de Daniella olivieri (11,1\%).

Les principales parties des plantes utilisées dans la préparation de ces remèdes sont les feuilles (56,2\%) et les écorces (25\%). Ces parties sont récoltées le jour, notamment au cours de la matinée, parfois très tôt avant le levé du soleil. Les instruments de récolte sont constitués de pioches, de coupe-coupes et de couteaux.

Les formes galéniques des remèdes les plus utilisées par les tradipraticiens sont la macération, la décoction et la poudre à incorporer dans la ration de l'animal (resp. $27,7 \%$ pour chacun). Les opérations de macération et de décoction sont effectuées dans des canaris en argile ou dans des calebasses. A l'issue de ces préparations, la solution à administrer à l'animal est filtrée avec un tissu propre avant d'être utilisée. A chaque répétition du traitement, la même procédure de préparation est renouvelée.

L'administration des remèdes se fait principalement par la voie orale. La durée des traitements varie d'un (1) à sept (7) jours en fonction de la recette. La posologie des recettes est également variable et dépend de la forme galénique. Le coût de la consultation et de la préparation des recettes est gratuit (94\%) ou se résume à une contribution symbolique (piécettes ou quelques noix de colas) (6\%). 
Tableau 1 : Recettes traditionnelles préconisées par les tradipraticiens dans le traitement des parasitoses digestives des petits ruminants

\begin{tabular}{|c|c|c|c|}
\hline \multicolumn{3}{|l|}{ Plantes ou produits utilisés } & \multirow[t]{2}{*}{ Organes, modes de préparation et voies d'administration } \\
\hline Langues locales ou nature du produit & Espèces & Familles & \\
\hline Welba (M) & Loranthus sp. & Loranthaceae & $\begin{array}{l}\text { Feuilles, macération et ajout de piments ; per os } 2 \text { fois/jour (matin et soir) pendant } 4 \\
\text { jours. }\end{array}$ \\
\hline Rambzoingo & Gardenia erubescens & Rubiaceae & Feuilles, macération ; per os 2 fois/jour (matin et soir) pendant 4 jours. \\
\hline Yilga (M), Gomgomais (P) & Mitragyna inermis & Rubiaceae & Feuilles, décoction ; per os 2 fois/jour (matin et soir) pendant 7jours. \\
\hline Bleu de méthylène & - & - & $\begin{array}{l}\text { A mélanger dans un litre d'eau de boisson ; deux cuillérées à soupe per os par jour } \\
\text { jusqu'à guérison. }\end{array}$ \\
\hline Gompelega (M) & Acacia raddiana & Mimosaceae & Ecorce, macération ; per os le matin en une seule prise. \\
\hline Kouka (M) & Khaya senegalensis & Meliaceae & Ecorce, macération ; per os le matin en une seule prise. \\
\hline Kaolin et charbon de bois & - & - & $\begin{array}{l}\text { Deux boules de kaolin à écraser et à mélanger à une quantité égale de charbon de bois. } \\
\text { Ensuite, ajouter un peu d'eau et mélanger à l'aliment servir per os. }\end{array}$ \\
\hline Gouinga (M) & Ceiba pentandra & Bombacaceae & Racine, macération ; per os en une seule fois. \\
\hline Pelèga $(\mathrm{M})$ & $\begin{array}{l}\text { Securidaca } \\
\text { longepedunculata }\end{array}$ & Polygalaceae & Racine, décoction ; per os 2 fois/jour (matin et soir) pendant 3 jours. \\
\hline Banguena (M) & Piliostigma reticulatum & Caesalpiniaceae & $\begin{array}{l}\text { Feuilles, pilées et mises dans l'eau de boisson dans laquelle } 3 \text { piments sont ajoutés et } \\
\text { servir per os matin et soir pendant } 4 \text { jours. }\end{array}$ \\
\hline Silsako (M) & - & - & Feuilles, pilées et mélangées à l’aliment ; per os en une seule fois. \\
\hline Papayer & Carica papaya & Caricaceae & $\begin{array}{l}\text { graines séchées, écrasées et mélangées à l’aliment ; per os dans une petite boîte de } \\
\text { tomate/jour/adulte pendant } 2 \text { jours. }\end{array}$ \\
\hline Siiga (M), Kojoli (P) & Anogeissus leiocarpus & Combretaceae & $\begin{array}{l}\text { - Fruits ou feuilles, écrasé(e)s et mélangé(es) au son de mil ; per os } \\
\text { - Fruits ou feuilles, écrasé(e)s et mélangé(es) aux céréales et y ajouter de la potasse; per os } \\
\text { - Feuilles, décoction ; per os } 1 \text { fois/jour (matin) pendant } 3 \text { jours. }\end{array}$ \\
\hline Dooki (P) & Combretum glutinsum & Combretaceae & Fruits ou feuilles, écrasé(e)s et mis(es) dans de l'eau de boisson ; per os \\
\hline Kaonga /Aonga (M) + Siiga (M) & $\begin{array}{l}\text { Daniellia oliveri }+ \\
\text { Anogeissus leiocarpus }\end{array}$ & $\begin{array}{l}\text { Caesalpiniaceae et } \\
\text { Combretaceae }\end{array}$ & Ecorce et feuilles, décoction, sel ; per os en une seule prise. \\
\hline Aonga (M) & Daniellia oliveri & Caesalpiniaceae & Ecorce, décoction ; per os en une fois par jour pendant 3 jours. \\
\hline
\end{tabular}

M : langue Morée $\quad \mathrm{P}$ : langue Peul 


\section{DISCUSSION}

Pour les soins de santé des animaux dans notre région d'étude, les éleveurs ont concomitamment recours à la médecine vétérinaire moderne et à la médecine traditionnelle. Cette médication traditionnelle fondée sur les savoirs endogènes du terroir et appliquée à la gestion de la bonne santé des animaux est faite essentiellement à base de plantes, comme l'ont mentionné Tamboura et al. (1998) et CAPES (2006). Elle est dispensée par des personnes relativement âgées et investies d'une certaine confiance de la part des éleveurs et agro-pasteurs du milieu dans lequel ils vivent. Un grand nombre d'éleveurs font encore confiance en ces techniques et savoirs endogènes comme l'ont déjà évoqué Tamboura et al. (1998) et CAPES (2006) au Burkina Faso, Barry et al. (2001) en Guinée Conakry, ainsi que HounzangbéAdoté (2005) qui estime leur proportion autour de $40 \%$ au Bénin.

Dans notre étude, les raisons qui poussent les uns et les autres à recourir à la médecine vétérinaire traditionnelle sont nombreuses et variées, tenant à la situation et à l'expérience individuelle de chaque éleveur. Ainsi, à l'instar de Fajimi et Taiwo (2005), certains éleveurs ont remarqué que de plus en plus de molécules modernes sont peu efficaces contre les maladies qu'elles sont sensées traiter et d'autres évoquent le renchérissement exponentiel des prix pratiqués par les officines. Mais parmi toutes ces raisons, le motif le plus fondamental semble être le manque d'agent vétérinaire dans les villages et/ou leur indisponibilité au moment opportun. Cette remarque est observée également par Guèye (2002) chez les aviculteurs traditionnels au Sénégal. Concernant l'inefficacité de certaines molécules antiparasitaires évoquée par les éleveurs, des études de contrôle d'efficacité des produits médicamenteux conventionnels disponibles sur le marché pourrait être envisagés en vue de vérifier leur assertion. Enfin, les coûts élevés des produits antiparasitaires conventionnels évoqués par les éleveurs sont probablement le fait des vétérinaires modernes privés qui ont été encouragés à s'installer en clientèle par l'État. Par essence, ce sont des entreprises économiques qui ont une obligation de résultats bénéfiques pour faire faces à leurs multiples charges de fonctionnement. C'est donc tout cet ensemble qui pourrait expliquer le regain d'intérêt pour la médecine vétérinaire traditionnelle dans le pays en général, et spécialement dans la zone étudiée.

Les résultats de cette étude ont par ailleurs confirmé l'existence de tradipraticiens spécialisés dans le traitement des maladies animales en générale et celui des parasitoses digestives des petits ruminants en particulier. Cette observation mériterait d'être explorée pour lutter contre d'autres pathologies particulières (reproduction, infectieuses, etc.) en vue de valoriser les plantes médicinales au profit de la communauté des éleveurs ruraux.

Dans notre étude, l'oralité reste également le moyen essentiel de conservation et de transmission de connaissances des plantes médicinales au sein du lignage, confirmant les observations rapportées par Tamboura et al. (1998) et Barry et al. (2001), respectivement dans la province du Passoré au Burkina Faso et au Fouta Djallon (Guinée Conakry). Si l'on considère l'âge avancé des personnes-ressources assurant ces prestations, il faut craindre qu'à terme, il n'y ait de graves déperditions dans le contenu de ces savoirs lorsque l'on passe de génération en génération. En effet, dans notre étude, les jeunes s'intéressent peu à ces alternatives tradithérapeutiques et des tradipraticiens refusent de partager leurs connaissances ancestrales qu'ils conservent jalousement.

L'absence de femmes comme tradipraticienne dans notre échantillon pourrait s'expliquer par le fait que dans la zone concernée par notre étude, elles sont rarement propriétaires d'animaux.

L'analyse des tradipraticiens enquêtés sur les parasitoses digestives des petits ruminants conforte dans l'idée qu'ils ont une claire perception de la relation entre les vers et les symptômes décrits. En effet, les observations recueillies concordent bien avec les conclusions scientifiques de Belem et al. (2005a et 2005b) qui ont noté une phase d'hypermétabolisme parasitaire de juin à octobre et une autre d'hypométabolisme ou d'hypobiose larvaire de novembre à mai dans le même contexte écologique que notre étude.

Pour les tradipraticiens enquêtés, les symptômes décrits sont synonymes de mauvaise santé chez l'animal qui les extériorise à travers les modifications de son 
comportement normal ou de son état général. $\mathrm{La}$ survenue de tels signes doit immédiatement faire l'objet d'une intervention à but thérapeutique approprié pour éviter la mortalité du jeune sujet et/ou la baisse des performances zootechniques au niveau des adultes.

Il est commun que les remèdes utilisés soient composés majoritairement de végétaux rencontrés dans le terroir plus ou moins proche du tradipraticien comme rapporté par Hounzangbé-Adoté (2005), Githiori et al. (2005) et Koné et al. (2005). Ces remèdes sont préparés au moment de l'emploi ou très peu de temps avant. Cela est probablement à mettre en rapport avec les difficultés de conservation du produit dans le contexte villageois (absence de chaîne de froid) et les conditions hygiéniques de préparation (hygiène des intrants, des récipients, des mains du préparateur). En effet, les températures ambiantes élevées du milieu associées à l'important microbisme de l'environnement de préparation du remède conduisent au développement des moisissures et d'autres germes qui pourraient compromettre leur efficacité et les rendre plutôt dangereux.

En dehors de Pilostigma reticulatum, de Loranthus sp., de Combretum glutinsum et de Gardenia erubescens, les plantes à effet anthelminthique citées par les tradipraticiens de l'étude sont en accord avec les observations faites dans la sous région OuestAfricaine (tableau 2) où certaines plantes identifiées ont déjà été testées scientifiquement. L'utilisation de certains organes des plantes plutôt que d'autres ou la récolte à des moments bien précis au cours de la journée, traduisent la preuve que les tradipraticiens ont une excellente connaissance de la physiologie végétale, des organes-sites de biosynthèse des molécules actives et du moment de leur élaboration dans la plante (Bitsindou, 1996).

Tableau 2 : Plantes à effet anthelminthique utilisées par les éleveurs en médecine traditionnelle vétérinaire dans la sous région Ouest -Africaine

\begin{tabular}{|c|c|c|c|c|c|}
\hline $\begin{array}{l}\text { Plantes } \\
\text { médicinales }\end{array}$ & $\begin{array}{l}\text { Parties } \\
\text { utilisées }\end{array}$ & $\begin{array}{l}\text { Espèce animale } \\
\text { traitée }\end{array}$ & Type de parasite & Testée/citée & Références, pays \\
\hline \multirow[t]{4}{*}{$\begin{array}{l}\text { Anogeissus } \\
\text { leiocarpus }\end{array}$} & \multirow[t]{2}{*}{ - écorce } & - ruminants & - parasites internes & - citée & $\begin{array}{l}\text { - Tamboura et al. } \\
\text { (1998), Burkina Faso }\end{array}$ \\
\hline & & - in vitro & $\begin{array}{l}\text { - Haemonchus } \\
\text { contortus }\end{array}$ & - testée & $\begin{array}{l}\text { - Koné et al. (2005), } \\
\text { Côte d'Ivoire }\end{array}$ \\
\hline & $\begin{array}{l}\text { - écorce et } \\
\text { graines }\end{array}$ & - rat & $\begin{array}{l}\text { - Nippostrongylus } \\
\text { braziliensis }\end{array}$ & - testée & $\begin{array}{l}\text { - Ibrahim et al. (1984), } \\
\text { Nigéria }\end{array}$ \\
\hline & $\begin{array}{l}\text { - écorce et } \\
\text { tige }\end{array}$ & $\begin{array}{l}\text { - veaux de } 6 \text { à } 24 \\
\text { mois }\end{array}$ & - parasites internes & - citée & $\begin{array}{l}\text { - Tall (1994), } \\
\text { Mauritanie }\end{array}$ \\
\hline $\begin{array}{l}\text { Mitragyna } \\
\text { inermis }\end{array}$ & écorce de tige & $\begin{array}{l}\text { animaux } \\
\text { domestiques }\end{array}$ & helminthes & citée & Assogba (1984), Bénin \\
\hline \multirow[t]{4}{*}{$\begin{array}{l}\text { Khaya } \\
\text { senegalensis }\end{array}$} & - feuille & $\begin{array}{l}\text { - animaux } \\
\text { domestiques }\end{array}$ & - helminthes & - citée & $\begin{array}{l}\text { - Assogba (1984), } \\
\text { Bénin }\end{array}$ \\
\hline & \multirow[t]{3}{*}{ - écorce } & $\begin{array}{l}\text { - animaux } \\
\text { domestiques }\end{array}$ & - parasites internes & - citée & $\begin{array}{l}\text { - Somopogui (1998), } \\
\text { Guinée }\end{array}$ \\
\hline & & - ovins & $\begin{array}{l}\text { - nématodes } \\
\text { digestifs }\end{array}$ & - testée & $\begin{array}{l}\text { - Ademola et al. } \\
\text { (2004), Nigéria }\end{array}$ \\
\hline & & - bœufs et moutons & - parasites internes & - citée & $\begin{array}{l}\text { - Aniyéré (1994), } \\
\text { Tchad }\end{array}$ \\
\hline Carica papaya & graines & ovins & strongles digestifs & testée & $\begin{array}{l}\text { Hounzangbé-Adoté } \\
\text { (2005), Bénin }\end{array}$ \\
\hline Ceiba pentandra & feuilles & - équins et asins & parasites internes & citée & $\begin{array}{l}\text { Tall (1994), } \\
\text { Mauritanie }\end{array}$ \\
\hline Acacia raddiana & écorce & bétail & vers intestinaux & citée & Aniyéré (1994), Tchad \\
\hline Daniella oliveri & écorce & $\begin{array}{l}\text { animaux } \\
\text { domestiques }\end{array}$ & vers adultes & citée & $\begin{array}{l}\text { Carrière (2000), } \\
\text { Guinée }\end{array}$ \\
\hline \multirow[t]{2}{*}{$\begin{array}{l}\text { Securidaca } \\
\text { longepedunculata }\end{array}$} & - écorce & - In vitro & $\begin{array}{l}\text { - Strongyloides } \\
\text { papillosus }\end{array}$ & - Testée & $\begin{array}{l}\text { - Oussoumanou et } \\
\text { al.(1991), Sénégal }\end{array}$ \\
\hline & $\begin{array}{l}\text { - racines et } \\
\text { écorce de tige }\end{array}$ & - In vitro & $\begin{array}{l}\text { - Haemonchus } \\
\text { contortus }\end{array}$ & - Testée & $\begin{array}{l}\text { - Koné et al. (2005), } \\
\text { Côte d'Ivoire }\end{array}$ \\
\hline
\end{tabular}




\section{Conclusion}

Cette étude menée dans le plateau central du Burkina Faso confirme que la pharmacopée vétérinaire est une pratique qui est encore d'actualité pour un grand nombre d'éleveurs au Burkina Faso. Elle renferme d'importantes potentialités à exploiter en complément de la médecine vétérinaire moderne pour lutter contre les maladies animales en général et les parasitoses digestives en particulier. Dans ce sens, il parait opportun de mener des recherches expérimentales pour valider l'efficacité des recettes proposées en vue de leurs utilisations ultérieures dans un cadre sécurisé pour les éleveurs.

Pour l'heure, les services d'élevage des provinces enquêtées devraient s'investir à établir un climat de confiance avec les tradipraticiens en vue de les organiser dans un cadre formel propice à la valorisation de la pharmacopée vétérinaire. Dans ce sens, la connaissance des savoirs naturalistes endogènes des éleveurs pourrait être intégrée dans les schémas de développement intégré et participatif pour la préservation de la diversité biologique et la santé des animaux. Pour cela, des jardins botaniques de plantes médicinales (communautaires ou individuels) pourraient être vulgarisés au niveau des producteurs. La protection des essences médicinales en dépend, de même que l'écologie et la survie des populations du milieu.

\section{Références bibliographiques}

Ademola IO, Faghemi BO, Idowu SO. 2004. Evaluation of the anthelmintic activity of Khaya senegalensis extract against gastrointestinal nematodes of sheep: in vitro and in vivo studies. Veterinary Parasitology, 122: 151-164.

Aniyéré F. 1994. Ethno-pharmacopée vétérinaire : vue du Tchad. In Métissages en santé animale de Madagascar à Haïti. Actes du séminaire d'éthnopharmacopée vétérinaire "KAGALA», un partage de savoirs, des 15-22 avril 1993, Ouagadougou, Burkina Faso, Kasonia K, Ansay M (eds). Presses Universitaires de Namur/CTA/ACCT ; 223-228.

Assogba MN. 1984. Quelques enquêtes sur la pharmacopée traditionnelle vétérinaire en République du Bénin. Communication faite à la 13è conférence de la Société Ouest Africaine de Pharmacologie les 2324-25 Février à Cotonou.

Barry MS, Oularé K, Diallo A, Camara Y, Camara M, Baldé MA. 2001. La medicine traditionnelle au Fouta Djallon: République de Guinée. Revue Méd. Pharm. Afr., 15: 95-102.

Belem AMG, Kaboré A, Bessin R. 2005a. Gastrointestinal helminthes of sheep in the central, eastern and northern parts of Burkina Faso. Bull Anim. Hlth. Prod. Afr., 53: 13-23.

Belem AMG, Kaboré A, Bessin R. 2005b. Variations saisonnières des helminthes gastro-intestinaux chez la chèvre du plateau central du Burkina Faso. Revue Elev. Méd. Vét. Pays trop., 58(1-2): 3743.

Bitsindou M. 1996. Enquêtes de phytothérapie traditionnelle à Kindamba et Odzala (Congo) et analyse des convergences d'usage des plantes médicinales en Afrique Centrale. Thèse de Doctorat, Université Libre de Bruxelles, 482 p.

Carrière M. 2000. Flore de Guinée : appellations vernaculaires et usages traditionnels de quelques plantes. http://perso.orange.fr/a.i.r.e./guilex3.htm (le15/10/2006).

CAPES (Centre d'Analyse des Politiques Economiques et Sociales). 2006. Etat des lieux des savoirs locaux au Burkina Faso. Inventaire des bonnes pratiques et propositions pour leur contribution au développement. CAPES: Ouagadougou ; 448 pages.

Fajimi AK, Taiwo AA. 2005. Herbal remedies in animal parasitic diseases in Nigeria: a review. African Journal of Biotechnology, 4(4): 303-307.

Githiori JB, Hôglund J, Waller PJ. 2005. Ethnoveterinary plant preparations as livestock dewormers : practices, popular beliefs, pitfalls and propects for the future. Animal Health Research Reviews, 6(1): 91-103.

Guèye EF. 2002. Newcastle disease in family poultry: prospects for its control through ethnoveterinary medicine. Livestock Research for Rural Development, 14(5), http:/www.cipav. org.co/lrrd/lrrd14/5/guey145.htm 
Hounzangbé-Adoté SM. 2005. Propriétés anthelminthiques de 4 plantes tropicales testées in vitro et in vivo sur les nématodes gastro-intestinaux chez les petits ruminants Djallonké. Thèse de Doctorat, Université d'Abomey-Calavi, Benin, $240 \mathrm{p}$.

Ibrahim MA. Nwude N, Ogunsuni RA, Aliu YO. 1984. Screening of West African plants for anthelminthic activity. Livestock Systems Research in Nigeria's Subhumid Zone. Proceedings of the second ILCA/NAPRI Symposium held in Kaduna/Nigeria, 29 october-2 november 1984.

INERA (Institut de l'Environnement et de Recherches Agricoles). 2002. Atelier national d'analyse et de validation des enjeux, contraintes et besoins de recherche de la filière lait dans les zones péri-urbaines et pastorales aménagées du Burkina Faso. Document de synthèse. Bobo-Dioulasso, 27-30 mai 2002, 35 pages + annexes.

Koné MW, Kamanzi AK, Traoré D, Betschart B. 2005. Anthelmintic activity of medicinal plants used in Northern Côte d'Ivoire against intestinal helminthiasis. Pharmaceutical Biology, 43(1): 72-78.

Krecek RC, Waller PJ. 2006. Towards of implementation of the "basket of option" approach to helminth parasite control of livestock: Emphasis on the tropics/ subtropics. Veterinary Parasitology, 139: 270-282.

Oussoumanou T, Diouf A, Temple RA et Daffe BM. 1991. Plantes de la pharmacopée sénégalaise. Etude in vitro de l'activité antiparasitaire de l'écorce des racines de Securidaca longepedunculata Fres (Polygalaceae). Rev. Med. Pharm. Afr., 5:43-47.

Somopogui AS. 1998. Médecine vétérinaire traditionnelle en République de Guinée (Note de synthèse des activités). FROM N’DAMA N¹, 2-6.

Tall AM. 1994. Synthèse des fiches sur la pharmacopée vétérinaire traditionnelle en Mauritanie. In Métissages en santé animale de Madagascar à Haïti. Actes du séminaire d'éthnopharmacopée vétérinaire "KAGALA», un partage de savoirs, des 15-22 avril 1993, Ouagadougou, Burkina Faso, Kasonia K, Ansay M (eds). Presses Universitaires de Namur/CTA/ACCT. 146-151.

Tamboura HH, Kaboré H, Yaméogo SM. 1998. Ethnomédecine vétérinaire et pharmacopée traditionnelle dans le plateau central du Burkina Faso : cas de la province du Passoré. Biotechnol. Agron. Soc. Environ., 2(3): 181-191. 